\title{
THE INTERNAL AUDIT AS A PART OF THE RISK MANAGEMENT PROCESS IN THE PUBLIC SECTOR ENTITIES
}

\author{
Professor Phd Eugeniu Turlea, ,E-mail:eturlea@yahoo.com \\ Lecturer PhD Aurelia Stefanescu, E-mail:stefanescu.aura@gmail.com \\ Academy of Economic Studies, Bucharest
}

\begin{abstract}
Analyzing risk from the perspective of internal audit is a part of the overall risk management task that internal auditing is systematically and methodically carrying out for the entity, using procedures of risk identification and evaluation. Given the existent differences of opinion regarding the integration of internal audit in the overall risk equation, the hereby constructive research identifies and explains the role of internal audit in the risk management process within the entity belonging to the public sector; pinpoints the risks coming up in the activity of the entities from the public sector, analyzes the ways in which the internal audit, through its specific procedure, contributes to the management of risks.
\end{abstract}

\section{The concept of risk in internal audit}

In general, a risk is a possibility of an unexpected event occurring or not at a certain moment, resulting from unexpected adverse phenomena.

In an economic understanding, a risk is an uncertain event or process that could probably generate losses in an activity or operation. Therefore, the risk can only be presented in relation to the objectives; the risk of not meeting the objectives affects the entity by causing a loss of focus on reaching success and preventing it to reach its objectives. Looking at risk through the scope of objectives, there are advantages and disadvantages that can be identified, named Opportunities and Threats in the model of risk analysis. The Threats are associated to events that have a negative impact on the objectives. The advantages are Opportunities which can be used, but also omitted or ignored, thus not exceeding expectations. Thus, integrating management in the equation of risk requires knowledge of when and where to deal with risk.

According to the Standards on Internal Audit, a risk is a possibility of occurrence of an event that has an impact on reaching the objectives. The risk needs to be identified and its effects need to be minimized or cancelled altogether. This approach is a part of the overall risk management task that internal auditing is systematically and methodically carrying out for the entity, using procedures of risk assessment and evaluation.

The factors that can act as sources of risk in audit are: the specifics of the investigated materials deriving from the specifics of the public entity where the audit is being carried; the quality of the definition of the entity being audited, the quality of the processes of creating, coordinating and controlling the systems which collect and process information throughout the data acquisition phase; the incorrect interpretation of the significance of certain aspects of the audited entity; the procedures applied by the auditor during the audit process, according to his/her experience and professionalism.

In literature there are various criteria regarding the classification of risks: the nature of activities, the specifics of the entities, the probability of occurrence of risks, the nature of the risks, a.o.

In the generic norms of internal public audit there is a minimal classification of risks in the following categories: 
- Organizational risks: missing formalism of procedures, missing definition of responsibilities, insufficient organization of human resources, missing or non updated documentation;

- Operational risks: intentional or unintentional omission of accounting transactions, improper archiving of documents, missing or inadequate control over high risk transactions;

- Financial risks: insecure payments, non detection of those transactions carrying a high financial risk;

In order to decide on the way to deal with risks it is necessary to classify them also according to the degree of exposure to risk. Many risks can be quantified, while others, such as those threatening the image or reputation can only be subjectively evaluated, based on quality indicators. The specialists recommend classifying risks in the following categories: low, medium and high risks. Thus, the manager has a first informational device on which to build an efficient controlling system, able to prioritize risks according to their financial and organizational impact, according to their probability of occurrence, according to the frequency of errors which, if cumulated, could significantly influence the entity's activity and achievements.

Special attention must be granted tot the attitude of entities toward risk. Thus, we can find tolerated risks, transferred risks, treated risks and terminated risks.

- Tolerated risks are usually those risks whose impact can be quantified and thus compared against the effort needed for dealing with them, usually by setting up efficient controlling methods; estimating that the later one is too high one could decide to tolerate the risk up to a calculated tolerance level;

- Transferred risks are those for which the manager has decided to accept the cost of transferring them to a third party, e.g. the frequent externalization of auxiliary activities for that the operational risks could be transferred to the third party;

- Treated risks are those for which the manager decides to implement the adequate controls to reduce their effects and maintain them to an acceptable and supportable level;

- Terminated risks are those risks associated with some activities that have been allowed to finalize in order to not interrupt the entity's activity. The most effective controls are being set up at the end of the risky activity, detailing them component by component and analyzing in detail each operation, in order to identify the risks to which the activity has been subjected and their financial impact.

Out of the multitude of risks one cannot overlook the risk of fraud, one that has long and often appeared in all areas of the public sector. The fraud is made up of a series of irregularities and illegal actions committed with the intention of cheating.

Naturally, the categories of risk presented are not exhaustive, as risks are dependent on the specific entity whose management is being audited.

Risk is a challenge that needs to be faced. The concept of administration on which the corporate governance relies on forces the management to identify risks which could affect the activity of the entity and deal with them if necessary.

\section{The role of the internal audit as a part of the risk management process in the public sector entities}

Risk management is the process of identifying, evaluating, managing and controlling potential events or scenarios in order to offer a reasonable reassurance as to the reaching of previously established objectives.

The internal audit makes a contribution to risk prevention through the examination and evaluation of the relevance and efficiency of the internal control system, given the exposure to risk of each field within the public sector. 
The internal audit plays a vital role in the process of risk management, however most of the responsibility lies with the entity's leadership. In order for the objectives to be reached, it needs to ensure that the risk management process is properly implemented and operated. The auditing committee is responsible for checking the existence of adequate processes acting effectively within the management of risks.

The internal audit has the role of assisting the entity's leadership and the audit committee by examining and evaluating the risk management processes applied by the leadership, checking their effectiveness and elaborating reports and recommendations for the improvement of risk management processes. Within the missions of consultancy or counseling, the internal auditors may support the entity to evaluate and implement a risk management system and of controlling mechanisms that can deal with the risks.

The mission of internal audit is to evaluate the inherent risks associated with the operations and informational systems of the public entity regarding: the reliability and integrity of financial and operational information, the efficiency and effectiveness of operations, the protection of assets, respecting rules and contracts. The internal audit contributes to the prevention of risks by examining and evaluating the relevance and efficiency of the internal controlling system, with regard to the exposure to risk of each field within the public sector.

The evaluation of the risks to which the entities of the public sector are being subjected consists of the following steps: identifying the auditable operations, identifying the threats, identifying the inherent risks associated to the auditable operations, establishing the risk analysis criteria and their levels, assessing risks by: establishing the risk levels according to the given criteria, classifying and sorting the operations according to the associated risks.

In order to express an opinion on the adequacy of the risk management process, the internal auditors must make sure that the risk management process meets the following objectives: the risks incurring from the entity's strategy and activities are identified and classified; the management and Board have established the acceptable level of risk for the entity, including those strategically accepted risks of the entity, the activities of lowering the risk levels are created and implemented according to the levels set out by the management and Board; the permanent monitoring of activities is done for the purpose of periodic re-evaluation of risks and of the management measures; the progress reports of the risk management processes are periodically being sent to the management and Board and finally, the corporate governance processes must periodically offer a presentation of the risks, risk controlling strategies to those involved.

The role of the internal audit in the risk management process can evolve in time and can be in the following states: no intervention of the internal audit; the auditing of the risk management process within the internal audit program; the active and continuous support; the participation in the risk management process, particularly in supervisory committees and in reporting activities on this topic; and finally, coordinating the risk management process.

The entity's management and the audit committee have the mission of determining the role of the internal audit in the risk management process. The vision of the entity's management on the topic will differ according to the entity's culture, the competence of the internal auditors, the country's specific practices.

\section{Conclusions}

By identifying and evaluating the exposure to significant risks, but also by perfecting risk management systems, the internal audit helps to add value to the entities of the public sector, to limit the waste of resources, the likelyhood of fraud and corruption, to timely detect anomanies and defficiencies. The consequence of entities operating inefectively, inefficiently and not economically in the public sector with disregard to the legal boundaries and with deffective management are being felt within all of society in the form of: improper usage of available resources with negative 
effect on the objectives; running internal processes without worthy achievements, escalating social unrest as a result of subjective and unfounded decision making. With its highly specialized support, the audit provide the leverage needed to professionalize the management. On the other hand, the creditorrs, the Government and local administrations, the European Union, the International Monetary Fund, the World Bank and the public are interested in the way in which management decisions generate results for the entities in the public sector and in the quality of the leadership process and in the way in which public funds are spent.

The economic transformations at national and international level expose the entities of the public sector to extra risks, thus raising a challenge for the internal audit.

Bibliography:

Dascalu D, Nicolae F, "Internal Audit in Public Institutions", Economica Publishing House, Bucharest, 2006

Morariu A, Turlea E. "Accounting-Financial Audit", Economica Publishing House, Bucharest, 2004

Renard J.

Theory and practice in internal audit, translation, Ministry of Public Finances Publishing House, Bucharest, 2002

Stefanescu A,

Turlea E.-reviewer

Le rôle de l'audit interne dans la gouvernance corporative et le management du risque dans les institution de credit, International Conference of Young Researchers, Chisinau, 2007

Turlea E.

Performance Audit, essential element of professionalizing the management of public sector entities, Annales Universitatis Apulensis, Series Oeconomica, Finante-Contabilitate nr.8/2006

Turlea E. Stefanescu A. The Financial Performance of Romanian Public Institutions -A Challenge At The Moment Of Integration, " The Balkan Countries 1st International Conference On Accounting and Auditing", Trakya University -Faculty of Economics and Administrative Sciences, Association of Accounting and Finance Academicians, Erdine, Turkey, 2007 